\title{
PRODUÇÃO DE FITOMASSA POR PLANTAS DE COBERTURA NO AGRESTE MERIDIONAL DE PERNAMBUCO
}

\author{
Vandson Felipe dos Santos1*; Lucas Figueira da Silva ${ }^{1}$; José Rômulo de Araújo Neto"; Vanilson Pedro da Silva; \\ Alexandre Tavares da Rocha'; Gilmara Mabel Santos"; \\ 1Universidade Federal Rural de Pernambuco, Unidade Acadêmica de Garanhuns, Avenida Bom Pastor, S/N, CEP 55292-270, \\ Garanhuns, PE. \\ *Fone (81)98922-3987, E-mail:vandsonfelipe19@gmail.com.
}

\begin{abstract}
RESUMO: Objetivou-se com este trabalho quantificar a produção de fitomassa seca de diferentes plantas de cobertura, nas condições edafoclimáticas do Agreste Meridional de Pernambuco.0 experimento foi realizado na Fazenda Experimental da UFRPE/UAG, obedecendo o delineamento experimental de blocos casualizados com quatro repetições e os seguintes tratamentos: T1- crotalária [Crotalaria spectabilis], T2Feijão de corda [Vigna unguiculata L.], T3- Guandu-anão [Cajanus cajan (L.) Millsp.], T4- Sorgo [Sorghum bicolor (L.) Moench], T5- Milheto [Pennisetum glaucum L.], T6- Milho [Zea mays L.]. Avaliou-se a altura da planta (AP), fitomassa verde (FV) e fitomassa seca (FS) no estádio de florescimento pleno das plantas. Os dados foram submetidos ao teste $F(P<0,05)$ e as médias comparadas pelo teste de Tukey a $5 \%$ de significância, utilizando-se o programa estatístico Sisvar. Os resultados mostraram que o sorgo destacouse entre todas as plantas de cobertura apresentando a maior quantidade de FV e FS e maior altura das plantas. Entre as leguminosas a crotalária apresentou a maior quantidade de FV e FS seguida do guandu. Assim, essas culturas apresentam-se como boas alternativas para a produção de palhada nas condições edafoclimáticas do estudo.
\end{abstract}

PALAVRAS-CHAVE: palhada, gramíneas, leguminosas.

\section{PHYTOMASS PRODUCTION BY COVER PLANTS IN THE SOUTHERN AGRESTE OF PERNAMBUCO}

\begin{abstract}
The objective of this work was to quantify the production of dry mass of different cover species, in the edaphoclimatic conditions of the Southern Agreste of Pernambuco. The experiment was carried out at the Experimental Farm of UFRPE/UAG. The experimental design of randomized blocks with four replications and the following treatments: T1 - crotalaria [Crotalaria spectabilis], T2 - String bean [Vigna unguiculata L.], T3 - Guandu [Cajanus cajan (L.) Millsp.], T4 - Sorghum [Sorghum bicolor (L.) Moench], T5 - Millet [Pennisetum glaucum L.], T6 - Corn [Zea mays L.] The Plant height (AP), green mass (VF) and drymass (FS) were evaluated at the full flowering stage of the plants. The data were submitted to the $F$ test $(P<0.05)$ and the means compared by the Tukey test at $5 \%$ significance, using the statistical program Sisvar. The results showed that alternatives for straw production in the edaphoclimatic conditions of the study. The sorghum stood out among all the cover plants presenting the largest amount of FV and FS and the highest height of the plants. Among the legumes, the crotalaria presented the highest amount of FV and FS followed by the guandu.
\end{abstract}

KEY WORDS: no-till, straw, legumes.

\section{INTRODUÇÃO}

Aregião do Agreste Meridional de Pernambuco possui uma produção agropecuária diversificada, destacando-se as criações de bovinos e os cultivos de milho, feijão e mandioca (Monteiro et al., 2007). Na maioria das propriedades adota-se o sistema de cultivo tradicional e em sequeiro, com a produção concentrada no período chuvoso. Assim, após a colheita os solos permanecem descobertos até o próximo ano agrícola. Isso, aliado as irregularidades pluviométricas e a baixa fertilidade natural dos solos (Santos et al., 2012), contribuem para o processo 
erosivo e o declínio da produtividade das lavouras na região.

Nas últimas décadas tem-se adotado no Brasil sistemas de manejo como o plantio direto e a adubação verde que visam reduzir os impactos da agricultura sobre os solos, utilizando-se restos culturais na superfície para proteção do terreno contra a erosão e para melhorar a fertilidade das camadas do solo (Cruz, 2006). Assim, o cultivo de plantas de cobertura exclusivamente para produção de palhada torna-se uma boa alternativa para 0 Agreste.

As plantas de cobertura além de protegerem o solo dos agentes climáticos, sequestram o carbono atmosférico e apresentam potencial para manter ou elevar o teor de matéria orgânica, mobilizar e reciclar nutrientes (Silva et al., 2011).

A palhada dessas das plantas de cobertura sobre a superfície do solo funciona como um reservatório de nutrientes, que são disponibilizados no decorrer do processo de decomposição, mediante a ação dos microrganismos presentes no solo (Costa et al., 2015). Entretanto, para manter a eficiência desse sistema de cultivo é imprescindível a escolha correta das plantas de cobertura, preconizando aquelas adaptadas as condições edafoclimáticas da região e que apresentem elevadas produtividades de fitomassa seca e uma boa cobertura do solo.

Objetivou-se com este trabalho quantificar a produção de fitomassa seca de diferentes plantas de cobertura, nas condições edafoclimáticas do Agreste Meridional de Pernambuco.

\section{MATERIAL E MÉTODOS}

0 experimento foi realizado na Fazenda Experimental pertencente à Universidade Federal Rural de Pernambuco/Unidade Acadêmica de Garanhuns (UFRPE/UAG), no município de Garanhuns-PE, localizada na latitude 08 $58^{\prime} 28^{\prime \prime}$ $\mathrm{S}$ e longitude de $36^{\circ} 27^{\prime} 11^{\prime \prime} \mathrm{O}$, altitude de $736 \mathrm{~m}$, no período de junho a outubro de 2017. A região apresenta predominância de um clima mesotérmico tropical de altitude (Cs'a), segundo a classificação de Köppen (Melo e Almeida, 2013) com precipitação pluviométrica de $897 \mathrm{~mm}$ anuais (Borges Júnior et al., 2012), compreendida entre os meses de maio a agosto.
0 delineamento experimental obedeceu ao sistema de blocos casualizados (DBC) com quatro repetições e os seguintes tratamentos: T1 - crotalária [Crotalaria spectabilis], T2 - Feijão de corda [Vigna unguiculata L.], T3 - Guandu-anão [Cajanus cajan (L.) Millsp.], T4 - Sorgo [Sorghum bicolor (L.) Moench], T5 - Milheto [Pennisetum glaucum L.], T6 - Milho [Zea mays L.]. Cada espécie foi estabelecida em semeadura manual no dia 10/06/2017 em linha com densidade de plantas e espaçamento realizados de acordo com a recomendação técnica para cada cultura (Cruz, 2010; Ferreira, et al. 2016; Ribeiro, 2002). A adubação foi realizada conforme a recomendação estadual de fertilizantes e corretivos (Cavalcanti, 2008).

A avaliação foi realizada de acordo com o ciclo de cada cultura, no estádio de florescimento pleno das plantas. As variáveis avaliadas foram: Altura da Planta (AP), Fitomassa Verde (FV) e Fitomassa Seca (FS). Para avaliação da altura, foi usada uma fita milimetrada, medindo-se a altura de 10 plantas aleatórias, a partir da superfície do solo até 0 ápice. Para avaliação da fitomassa da crotalária, feijão de corda, guandu e milho, foram coletadas dez plantas aleatórias na área útil de cada tratamento. Para o milheto e sorgo foi retirada uma amostra aleatória de $0,375 \mathrm{~m} 2$. O corte das plantas foi realizado rente à superfície do solo. Todo o material verde foi pesado em campo, para determinação da FV, em seguida, foram levados para secagem em estufa a $65^{\circ} \mathrm{C}$ até estabilização da massa, para determinação da FS, sendo as quantidades extrapoladas em $\mathrm{kg} \mathrm{ha}^{-1}$.

Os dados foram submetidos ao teste $F$ $(\mathrm{P}<0,05)$ da análise de variância, e as médias de tratamentos comparadas pelo teste de Tukey a $5 \%$ de significância, utilizando-se o programa estatístico Sisvar (Ferreira, 2000).

\section{RESULTADOS E DISCUSSÃO}

Os resultados evidenciaram uma superioridade das gramíneas sorgo, milho e milheto em relação às leguminosas como plantas de cobertura observando-se efeitos significativos nos parâmetros analisados (Tabela 1). Entretanto, as leguminosas apresentaram menores ciclos de produção, possibilitando o seu cultivo em sistemas de sucessão antes da cultura principal. 
TABELA 1. Resumo da análise de variância para a altura da planta $(\mathrm{cm})$, produção de fitomassa verde $\left(\mathrm{Mg} \mathrm{ha}^{-1}\right)$ e Fitomassa seca $\left(\mathrm{Mg} \mathrm{ha}^{-1}\right)$ de diferentes plantas de cobertura.

\begin{tabular}{|c|c|c|c|c|}
\hline \multirow{2}{*}{\multicolumn{2}{|c|}{$\begin{array}{l}\text { Tratamento } \\
\mathrm{Cm}\end{array}$}} & Altura da planta & Fitomassa Verde (FV) & Fitomassa Seca (FS) \\
\hline & & Mg ha-1 $^{-1}$ & $\mathrm{Mg} \mathrm{ha}^{-1}$ & \\
\hline Crotalaria & & $57,5^{d}$ & $17,05^{c b}$ & $3,70^{\circ}$ \\
\hline Feijão de corda & & $39,2^{\mathrm{e}}$ & $6,42^{\mathrm{e}}$ & $1,68^{\circ}$ \\
\hline Guadu & & $82,1^{\circ}$ & $8,68^{\mathrm{de}}$ & $2,95^{\circ}$ \\
\hline Milheto & & $144,1^{\mathrm{b}}$ & $22,7^{b}$ & $7,3^{b}$ \\
\hline Milho & & $137,7^{\mathrm{b}}$ & $14,67^{\mathrm{cd}}$ & $8,84^{b}$ \\
\hline Sorgo & & $167,9^{\mathrm{a}}$ & $38^{\mathrm{a}}$ & $11,5^{\mathrm{a}}$ \\
\hline $\mathrm{FV}$ & GL & & ANOVA- Teste F & \\
\hline Tratamento & 5 & $1,09^{* \star}$ & $523,78^{\star *}$ & $58,57^{* *}$ \\
\hline Bloco & 3 & 0,014 ** & $15,68^{\text {ns }}$ & $0,61^{\text {ns }}$ \\
\hline CV (\%) & & 7,57 & 16,33 & 16,95 \\
\hline
\end{tabular}

Médias seguidas de mesma letra, minúscula na coluna, não diferem entre si pelo teste de Tukey $(p<0,05) .{ }^{* *}$ : significativo a $5 \%$ de probabilidade pelo teste $F$; ns : não significativo pelo teste $F$.

Em relação a altura e a quantidade de fitomassa verde, a cultura do sorgo destacou-se entre as gramíneas, alcançando o maior tamanho e maior produção. A C. spectabilis apresentou a maior produção de FV, entretanto, a maior altura de plantas foi apresentada pelo feijão guandu. Por outro lado 0 $V$. uniculata apresentou as menores quantidade de matéria fresca e tamanho de planta. $O$ desempenho do feijão de corda foi prejudicado durante a condução do experimento em virtude de uma grande desfolha das plantas, onde esse processo pode estar relacionado a fatores bióticos e abióticos que afetam as plantas (Barbosa et al., 2013).

Entre as leguminosas não se observou diferenças significativas em relação a produção de matéria seca, com uma variação entre 1,68 e 3,7 Mg ha-1. Entretanto nota-se que a crotalária apresentou a maior quantidade de FS, seguido do guandu. Enquanto que o feijão de corda apresentou o menor valor de FS. A utilização de leguminosas como plantas de cobertura é bastante interessante pela sua capacidade de fixação biológica de nitrogênio (Rossi et al., 2013), possibilitando ciclagem mais eficiente de $\mathrm{N}$ no sistema de produção.

Entre as gramíneas o S. bicolor apresentou a maior produção de FS seguido do milho com 11,5 e 8,84 Mg ha-1 respectivamente, enquanto o milheto apresentou a menor quantidade de matéria seca na floração. $O$ sorgo é uma excelente opção como cultura de cobertura e produtora de palhada, em razão da sua resistência às condições de déficit hídrico, com elevada capacidade de aproveitamento da água e produção de matéria seca (Magalhães et al., 2001).

As massas de matéria seca do sorgo foram superiores aos observados por Teixeira et al., (2011) ao avaliarem a taxa de decomposição e a liberação de nutrientes de plantas de milheto e sorgo, cultivado em Seropédica no Estado do Rio de janeiro. Esses autores verificaram valores de $2500 \mathrm{~kg} \mathrm{ha}^{-1}$ no estádio de floração e $3560 \mathrm{~kg} \mathrm{ha}^{-1}$ no estádio de maturação das plantas de sorgo. Os maiores valores observados neste estudo, em comparação com os obtidos por Teixeira et al. (2001), podem ser decorrentes do uso de adubação com N-P-K antes da semeadura do sorgo e da maior precipitação ocorrida durante 0 experimento.

Considera-se ideal uma quantidade de palhada mínima de $6000 \mathrm{~kg} \mathrm{ha}^{-1}$ para uma eficiente cobertura do solo no sistema de plantio direto, no entanto, ressaltam que esses valores possam sofrer variações conforme o tipo de planta a região de cultivo e das condições edafoclimáticas (Alvarenga et al., 2001) Assim, apenas as gramíneas superaram esses valores. Geralmente esses valores estão associados à região do cerrado.

O sorgo apresentou o melhor desempenho entre as gramíneas com maior produção de palhada para cobertura do solo. As leguminosas apresentaram valores de fitomassa seca inferiores às gramíneas, no entanto, o uso da crotalária e do guandu são alternativas interessantes na região devido ao ciclo curto e possibilidade de adubação verde. Logo, recomendase essas culturas como formadoras de palhadas no Agreste Meridional de Pernambuco. 
Estudos sobre o potencial de plantas de cobertura em regiões do Agreste Meridionais ainda são incipientes, portanto, são necessários novas pesquisas sobre o tema.

\section{AGRADECIMENTOS}

À Universidade Federal Rural de Pernambuco I Unidade Acadêmica de Garanhuns (UFRPE /UAG) e ao Programa de Educação Tutorial (PET) pelo apoio financeiro.

\section{REFERÊNCIAS BIBLIOGRÁFICAS}

Alvarenga, R. C; Rodrigues, J. A. S.; Santos, F. C.; Gontijo Neto, M. M.; Viana, M. C. M. Plantas de cobertura de solo para sistema plantio direto. Informe Agropecuário, 2011, 22, 208, 25-36.

Barbosa, H.D; Lima, H. E.; Smiderle, O. O. Efeito da remoção de folíolos em diferentes estádios fenológicos do Feijão-Caupi em Roraima. In: CONGRESSO NACIONAL DE FEIJÃO-CAUPI, 3., 2013, Recife. Anais... Recife: IPA, 2013.

Borges Júnior, J.C.F; Anjos, R. T.; Silva T. J. A.; Lima, J. R. S.; Andrade, C. L. T. Métodos de estimativa da evapotranspiração de referência diária para a microrregião de Garanhuns, PE. Revista Brasileira de Engenharia Agrícola e Ambiental, 2012, 16, 4, 380-390.

Cavalcanti F. J. A. Recomendações de adubação para o estado de Pernambuco: segunda aproximação. Instituto Agronômico de Pernambuco - IPA: Recife, Pernambuco, 2008, 198p.

Costa, N. R.; Andreotti, M.; Ulian, N. A.; Costa, B. S.; Pariz, C. M.; Cavasano, F. A.; Teixeira Filho, M. C. M. Produtividade da soja sobre palhada de forrageiras semeadas em diferentes épocas e alterações químicas no solo. Revista Brasileira de Ciências Agrárias, 2015, 10, 1, 8-16.

Cruz da, E. S. Influência do preparo do solo e de plantas de cobertura na erosão hídrica de um Argissolo Vermelho-Amarelo. Seropédica: UFRRJ, 2006, 58p. Dissertação (Mestrado em Agronomia) - Universidade Federal Rural do Rio de Janeiro, UFRRJ.
Cruz, J. C. Cultivo do milho. Sistema de produçãoPlantio. Embrapa Milho e Sorgo, $6^{\text {a }}$ ed, Sete Lagoas, Minas Gerais, 2010. Disponível em:<https://ainfo. cnptia.embrapa.br/digital/bitstream/item/27037/1/ Plantio.pdf>. Acesso em: 08 mar. 2019.

Ferreira, A. C. de B.; Bogiani, J. C.; Sofiatti, V.; Lamas, F. M. Sistemas de Cultivo de Plantas de Cobertura para a Semeadura Direta do Algodoeiro. Comunicado técnico 377, Embrapa Algodão-Campina Grande, 2016. 15p. Disponível em: <https://www.embrapa.br/algodao/ busca-de-publicacoes/-/publicacao/1066067/sistemasde-cultivo-de-plantas-de-cobertura-para-a-semeaduradireta-do-algodoeiro> Acesso em: 19 de jan. 2020.

Ferreira, P. V. Estatística experimental aplicada a agronomia. 3. ed. Maceió: EDUFAL, 2000. 422 p.

Magalhães, P.C.; Silva, J.B.; Durães, F.O.M.; Karam, D.; Ribeiro, L.S. Efeito de doses reduzidas de glyphosate e paraquat simulando deriva na cultura do sorgo. Revista Planta Daninha, 2001, 19, 2, 255-262.

Melo, F.P.; Almeida, J.P. Análise das feições geomorfológicas e dos processos morfodinâmicos do sítio urbano de Garanhuns-PE. Ambivalências - Revista do Grupo de Pesquisa "Processos Identitários e Poder" - GEPPIP, 2013, 1, 1, 1-12.

Monteiro, A. A.; Tamanini, R.; Silva, L. C. C.; Mattos, M. R.; Magnani, D. F.; d'Ovidio, L.; Nero, L. A.; Barros, M. A. F.; Pires, E. M. F.; Paquereau, B. P. D.; Beloti, V. Características da produção leiteira da região do agreste do estado de Pernambuco, Brasil. Semina: Ciências Agrárias, 2007, 28, 4, 665-674.

Ribeiro, V. Q. Cultivo do feijão-caupi (Vigna unguiculata (L.) Walp). Sistema de Produção. Embrapa MeioNorte: Teresina, Piauí, 2002, 108p. Disponível em: < https://www.embrapa.br/busca-de-publicacoes/-/ publicacao/66591/cultivo-do-feijao-caupi-vignaunguiculata-l-walp>. Acesso em: 19 de jan. 2020.

Rossi, C. Q.; Pereira, M G.; Giácomo, S. G.; Betta, M.; Polidoro, J. C. Decomposição e liberação de nutrientes da palhada de braquiária, sorgo e soja em áreas de plantio direto no cerrado goiano. Semina: Ciências Agrárias, 2013, 34, 4, 1523-1534. 
Santos, J. C. B.; Souza Júnior, V. S.; Corrêa, M. de fósforo em solos distintos. Semina: Ciências Agrária, M.; Ribeiro, M. R.; Almeida, M. C.; Borges, L. E. P. 2011, 32, 4, 1315-1326.

Caracterização de Neossolos Regolíticos da região semiárida de Pernambuco. Revista Brasileira de Ciência do Solo, 2012, 36, 3, 683-695.

Teixeira, M. B.; Loss, A.; Pereira, M.G.; Pimentel, C. Decomposição e liberação de nutrientes da parte aérea de plantas de milheto e sorgo. Revista Brasileira de Silva, T. O.; Furtini Neto, A. E.; Carneiro, L. F.; Paludo, C Ciência do Solo, 2011, 35, 3, 867-876.

$\checkmark$. Plantas de cobertura submetidas a diferentes fontes 